\title{
EDITORIAL
}

\section{SCREENING FOR THE ANOREXIA OF AGING}

\author{
C. WARNE ${ }^{1}$, I.T. FORRESTER ${ }^{2}$, L. JONES ${ }^{3}$, J.E. MORLEY ${ }^{4}$
}

\begin{abstract}
1. Department of Nutrition and Dietetics, Doisy College of Health Sciences, St. Louis, Missouri, USA; 2. Department of Nutrition and Dietetics, Doisy College of Health Sciences, St. Louis, Missouri, USA; 3. Department of Nutrition and Dietetics, Doisy College of Health Sciences, St. Louis, Missouri, USA; 4. Division of Geriatric Medicine, Saint Louis University School of Medicine, St. Louis, Missouri, USA. Corresponding author: John E. Morley, MB, BCh, Division of Geriatric Medicine, Saint Louis University School of Medicine, 1402 S. Grand Blvd., M238, St. Louis, MO 63104,Email: john.morley@health.slu.edu
\end{abstract}

Key words: Anorexia, aging, malnutrition, nutrition.

"I am grateful to old age because it has increased my desire for good conversation and decreased my desire for good food." $\sim$ M. Tuillus Cicero

It is now recognized that as people age, they decrease their food intake (1). This physiological change with aging is termed the "Anorexia of Aging" $(2,3)$. Physiological anorexia puts older people at increased risk for weight loss, malnutrition, and reduced quality of life $(4,5)$. Appetite and food intake have received little attention in health care and research because appetite is based on subjective experiences of an individual and is difficult to measure (6). The Global Leadership Initiative on Malnutrition (7) has 3 components for the diagnosis of malnutrition, namely non-volitional weight loss, low body mass index, and a reduction in muscle mass. The consensus stated that this could be due to a reduction of food intake or poor absorption and/or inflammation, and underlying disease as the causative factors.

A robust screening tool to evaluate appetite among older persons could create opportunities for early intervention among health care providers, and could potentially lessen healthcare costs. Numerous screening tools exist to diagnose protein energy malnutrition (8). Of these, the Mini Nutritional Assessment (MNA) and its short form, the Simplified Nutritional Appetite Questionnaire (SNAQ), have become the most widely used among older people (9-14). The SNAQ appears to be such a tool that is short, reliable, and validated with the ability to effectively and rapidly be used by health care professionals to assess appetite among older adults.

The 4-question SNAQ (Table 1) was originally derived from the longer Council on Nutrition Appetite Questionnaire (CNAQ). Wilson et al studied both the SNAQ and the CNAQ in 247 long term care and 709 community dwelling older adults. Their study concluded that both tools predict future weight loss, but that the SNAQ proved to be more efficient (5). Another study by Hanisah et al consisted of 145 hospitalized patients compared the CNAQ, the SNAQ, and the Hunger and Sensory Perception Questionnaire (AHSPQ). Using the Subjective Global Assessment, the study found 11\% of patients were severely malnourished, $50.4 \%$ were mild or moderately malnourished and $18 \%$ were normal (15). The study concluded that the SNAQ had the highest specificity and was more reliable and valid as an appetite screening tool than CNAQ among older adults.

In a cross-sectional study of 175 older individuals, the SNAQ was compared to the MNA; while the SNAQ did not predict malnutrition as classified by the MNA, the authors concluded that an abnormal SNAQ score may be effective in identifying individuals at risk of significant weight loss (16). Ilhan et al examined 442 persons over 60 years of age and found that the SNAQ had reliability of 0.693 and test-retest stability. It also had a moderate correlation with the MNA (17). A separate study found that the SNAQ predicted lower muscle mass among other factors of older people with poor appetite (18).

Additional studies have assessed effectiveness of SNAQ in more specific illness and disease settings, such as heart failure. More than 26 million people worldwide suffer from the complex clinical syndrome (19). Decreased appetite among heart failure patients contributes to the development of poor nutritional status, which may negatively influence prognosis. In a study of 186 heart failure patients, both the CNAQ and SNAQ were shown to have construct validity and internal consistency validity (14). Additionally, a separate study of 48 patients undergoing mitral valve surgery concluded the SNAQ showed significant appetite improvement after one year of recovery from surgery (20). Another study of 99 patients undergoing aortic valve replacement found the SNAQ was able to detect those who were considered to be moderately malnourished (21).

Another disease area thoroughly studied is gynecological cancer, which show increasing incidence; the disease now accounts for $19 \%$ of new female cancer cases worldwide. Among several symptoms, malnutrition may be a key contributor to poor clinical outcomes, specifically high mortality rates. Malnutrition affects 20 to $26 \%$ of individuals with gynecological cancers, specifically 50 to $67 \%$ of individuals with ovarian cancer (22). A cross-sectional study conducted in Korea described nutritional status and identified nutritional factors contributing to malnutrition. A total of 129 individuals over the age of 20 with gynecological cancers, including cervical, peritoneal, ovarian, uterine, endometrial, vaginal, and vulvar, were included. Using the SNAQ to assess nutritional status, the study concluded $53.5 \%$ of patients screened at risk of severe malnutrition, as classified by the 


\section{THE JOURNAL OF NUTRITION, HEALTH \& AGING@}

Patient-Generated Subjective Global Assessment (PG-SGA), indicating SNAQ to be an adequate nutritional assessment in gynecological cancers (23).

Other areas of study, including pulmonary disease and rehabilitation programs, have verified the validity and reliability of the Simplified Nutritional Appetite Questionnaire (SNAQ). A cross-sectional study of 229 individuals conducted in Australia evaluated nutritional status of older adults participating in ambulatory rehabilitation and determined the associations between nutritional status, physical function, and quality of life. Of those, $36 \%$ experienced a stroke, $19 \%$ had an orthopedic procedure, $45 \%$ had another medical condition. The study results showed the Mini Nutritional Assessment (MNA) detected $63 \%$ of individuals were malnourished or at risk of severe malnutrition and the SNAQ detected one-third at risk of $>5 \%$ weight loss in the subsequent six months. The study findings emphasized the validity of SNAQ to detect malnutrition among older adults (24). Additionally, a quasiexperimental study of nine patients in Malaysia evaluated a multi-disciplinary, eight-week pulmonary rehabilitation program and considered improvements of nutritional and functional status, as well as the quality of life of chronic obstructive pulmonary disease (COPD) out-patients. The study concluded the eight-week pulmonary rehabilitation program effectively improved functional status, specifically among men, as well as quality of life; however, the program did not impact nutritional status (25). The findings support the verification of SNAQ, but due to the small sample size and $50 \%$ completion rate, the results of this study should be interpreted with caution and therefore cannot be extrapolated to the general population of elderly.

Several additional studies have used SNAQ as a screening tool. In a population-based cross-sectional study of 672 people with a mean age of $71.7+6.54$ SNAQ detected $28.7 \%$ of the elderly to be at increased risk of weight loss (26). A study conducted in China among people with cirrhosis of the liver found SNAQ able to predict weight loss and a SNAQ score below 14 correlated with hemoglobin, albumin and pre-albumin (27). In a Japanese study of 316 residents from nursing homes, both the CNAQ and the SNAQ predicted one-year mortality (28). The Japanese versions of the CNAQ and SNAQ had previously been validated in a larger group (29). In an English hospital study, $42 \%$ had poor appetite using the SNAQ and this was predictive of hospital acquired infections and mortality (30). In ambulatory older persons the SNAQ compared to the MNA had a positive predictive value of $89 \%$ and a negative predictive value of $44 \%$ (31). In 84 community dwelling persons in Japan the SNAQ had reasonable psychometric properties and correlated with the MNA-short form, walking speed, chair stand, timed up and go test and the geriatric depression scale (32). SNAQ scores and calorie counts were lower in patients with chronic obstructive pulmonary disease exacerbations (33). Both increased at discharge from hospital.

Overall, this brief literature review gives credence to the concept that the SNAQ can identify older people with poor appetite who are at risk for future weight loss and other poor outcomes. The SNAQ has adequate psychometric characteristics and is included in the Rapid Geriatric Assessment, which has been developed for health providers to recognize the geriatric syndromes (Frailty (34), sarcopenia $(35,36)$, cognitive dysfunction $(37,38)$ and anorexia of aging $(39,40))$. The management of a low SNAQ score requires, at the minimum, exclusion of depression, medications, dysphagia, dental problems, Helicobacter pylori or other nosocomial infections, hypercalcemia, Addison's disease, inability to selffeed (e.g., tremors), cholecystitis and therapeutic diets as the causes of the anorexia (40). Persons with anorexia should also be encouraged to use calorie supplements one to two hours before meals (41). Identification and treatment of anorexia in older persons represents a key component of preventive care for geriatric patients.

Table 1

SNAQ (Simplified Nutritional Assessment Questionnaire)

Name:_ Sex: M F
Age: $:_{------}$Height:_ Weight:
Date:_-
Food tastes

a. very bad b. bad c. average d. good e. very good

\section{Normally I eat}

a. less than one meal a day. b. one meal a day. c. two meals a day. d. three meals a day. e. more than three meals a day.

\section{My appetite is}

a. very poor b. poor c. average d. good e. very good

\section{When I eat}

a. I feel full after eating only a few mouthfuls. b. I feel full after eating about a third of a meal.

c. I feel full after eating over half a meal. d. I feel full after eating most of the meal.

e. I hardly ever feel full.

Tally the results based on the following numerical scale: $a=1 ; b=2, c=3, d=4, e=5$. The sum of the scores for the individual items constitutes the SNAQ score. A SNAQ score of $<14$ indicates significant risk of at least $5 \%$ weight loss within six months. Wilson, et al. (6).

Disclosures: The authors declare there are no conflicts.

\section{References}

1. Jadczak AD, Visvanathan R. Anorexia of Aging - an updated short review. J Nutr Health Aging 2019;23:306-309.

2. Morley JE, Silver AJ. Anorexia in the elderly. Neurobiol Aging 1988;9:9-16.

3. Morley JE. Anorexia of ageing: A key component in the pathogenesis of both sarcopenia and cachexia. J Cachexia Sarcopenia Muscle 2017;8:523-526.

4. Cederholm T, Jensen GL, Correia MITD;GLIM Core Leadership Committee; GLIM Working Group. GLIM criteria for the diagnosis of malnutrition - a consensus report from the global clinical nutrition community. Clin Nutr 2019;38:1-9.

5. Hanisah R, Suzana S, Lee FS. Validation of screening tools to assess appetite among geriatric patients. J Nutr Health Aging 2012;16:660-665. 


\section{SCREENING FOR THE ANOREXIA OF AGING}

6. Wilson MG, Thomas DR, Rubenstein LZ, et al. Appetite assessment: Simple appetite questionnaire predicts weight loss in community-dwelling adults and nursing home residents. Am J Clin Nutr 2005;82:1074-1081.

7. Omran ML, Morley JE. Assessment of protein energy malnutrition in older persons, Part I: History, examination, body composition, and screening tools. Nutrition 2000;16:50-63.

8. Guigoz Y, Lauque S, Vellas BJ. Identifying the elderly at risk for malnutrition. The Mini Nutritional Assessment. Clin Geriatr Med 2002;18:737-757.

9. Vellas B, Villars H, Abellan G, et al. Overview of the MNA-its history and challenges. J Nutr Health Aging 2006;10:456-463.

10. Jukeran MS, Ritti-Dias RM, Franco FGM, et al. Nutritional risk by mini nutritional assessment (MNA), but not anthropometric measurements, has a good discriminatory power for identifying frailty in elderly people: Data from Brazilian secondary care clinic. J Nutr Health Aging 2019;23:217-220.

11. van Wissen J, van Stijn MF, Doodeman HJ, Joudijk AP. Mini nutritional assessment and mortality after hip fracture surgery in the elderly. J Nutr Health Aging 2016;20:964-968

12. Folven K, Biringer E, Abrahamsen JF. Mini nutritional assessment short-form (MNA-SF) predicts institutionalisation in an intermediate post-acute care setting. J Nutr Health Aging 2018;22:199-204.

13. Donini LM, Marrocco W, Marocco C, Lenzi A. Validity of the self-mini nutritional assessment (Self-MNA) for the evaluation of nutritional risk. A cross-sectional study conducted in general practice. J Nutr Health Aging 2018;22:44-52.

14. Andreae C, Stromberg A, Sawatzky R \& Arestedt K. Psychometric evaluation of two appetite questionnaires in patients with heart failure. J Cardiac Failure 2015;21:954 958.

15. Rolland Y, Perrin A, Gardette V, et al. Screening older people at risk of malnutrition or malnourished using the Simplified Nutritional Appetite Questionnaire (SNAQ): A comparison with the mini-nutritional assessment (MNA) tool. J Am Med Dir Assoc 2012;13:31-34

16. Kim J, Lee Y, Won CW, et al. Nutritional status and frailty in community-dwelling older Korean adults: The Korean frailty and aging cohort study. J Nutr Health Aging 2018;22:774-778.

17. Ilhan B, Bahat G, Oren MM, et al. Reliability and validity of Turkish version of the Simplified Nutritional Appetite Questionnaire (SNAQ). J Nutr Health Aging 2018;22:1039-1044.

18. Ilhan B, Bahat G, Erdogan T, Kilic C, Karan MA. Anorexia is independently associated with decreased muscle mass and strength in community dwelling older adults. J Nutr Health Aging 2019;23:202-206.

19. Saverese G, Lund LH. Global public health burden of heart failure. Cardiac Failure Review 2017;3(1); 7. Doi: 10.15420/cfr.2016:25:2.

20. Kim SA, Kang MK, Shim CY, et al. Changes of body composition after valve surgery in patients with mitral valve disease. PLoS One 2018;13:e0203798. Doi: 10.1371/ journal.pone.0203798. eCollection 2018.

21. Jaglielak D, Wernio E, Kozaryn R, et al. The impact of nutritional status and appetite on the hospital length of stay and postoperative complications in elderly patients with severe aortic stenosis before aortic valve replacement. Kardiochir Torakochirurgia Pol 2016;13:105-112

22. American Cancer Society. Cancer Facts \& Figures 2018. Atlanta: American Cancer Society; 2018

23. Nho JH, Kim SR, Kwon YS. Depression and appetite: Predictors of malnutrition in gynecological cancer. Support Care Cancer 2014;22:3081-3088. Doi.org/10.1007/ s00520-014-2340-y.

24. Kaur S, Miller MD, Halbert J, Giles LC, Crotty M. Nutritional status of adults participating in ambulatory rehabilitation. Asia Pacific J Clin Nutr 2008;17:199-207.

25. Suzana, Jr., Hanis M, Tang S, et al. Changes in nutritional, functional status and quality of life of COPD out-patients after a pulmonary rehabilitation programme in HUKM: A pilot study. Malaysia J Nutr 2008;14:151-162.

26. Acar Tek N, Karacil-Ermumcu MS. Determinants of health-related quality of life in home dwelling elderly population: Appetite and nutritional status. J Nutr Health Aging 2018;22:996-1002.

27. Wang T, Shen J. Usefulness of Simplified Nutritional Appetite Questionnaire (SNAQ) in appetite assessment in elder patients with liver cirrhosis. J Nutr Health Aging 2018;22:911-915.

28. Mikami Y, Watanabe Y, Edahiro A, et al. Relationship between mortality and council of nutrition appetite questionnaire scores in Japanese nursing home residents. Nutrition. 2019;57:40-45

29. Tokudome Y, Okumura K, Kumagai Y, et al. Development of the Japanese version of the Council on Nutrition Appetite Questionnaire and its simplified versions, and evaluation of their reliability, validity and reproducibility. J Epidemiol 2017;27:524 530 .

30. Pilgrim AL, Baylis D, Jameson KA, et al. Measuring appetite with the Simplified Nutritional Appetite questionnaire identifies hospitalized older people at risk of worse health outcomes. J Nutr Health Aging 2016;20:3-7.

31. Yaxlley A, Crotty M, Miller M. Identifying malnutrition in an elderly ambulatory rehabilitation population: Agreement between mini Nutritional Assessment and validated screening tools. Healthcare (Basel). 2015;3:822-829.

32. Nakatsu N, Sawa R, Misu S, Ueda Y, Uno R. Reliability and validity of the Japanese version of the simplified nutritional appetite questionnaire in community-dwelling older adults. Geriatr Gerontol Int 2015;15:1264-1269.

33. Wang Y, Shen Y, Zuo Q, et al. Evaluation of ghrelin level and appetite regulation in patients with acute exacerbations of chronic obstructive pulmonary disease. Int J Chron Obstruct Pulmon Dis 2014;9:863-870.

34. Morley JE, Vellas B, van Kan G, et al. Frailty consensus: A call to action. J Am Med Dir Assoc 2013;14:392-397.

35. Dent E, Morley JE, Cruz-Jentoft AJ, et al. International clinical practice guidelines for sarcopenia (ICFSR): Screening, diagnosis and management. J Nutr Health Aging 2018;22:1148-1161.

36. Malmstrom TK, Miller DK, Simonsick EM, et al. SARC-F: A symptom score to predict persons with sarcopenia at risk for poor functional outcomes. J Cachexia Sarcopenia Muscle 2016;7:28-36.

37. Malmstrom TK, Voss VB, Cruz-Oliver DM, et al. The rapid cognitive screen (RCS): A point-of-care screening for dementia and mild cognitive impairment. J Nutr Health Aging 2015;19:741-744.

38. Morley JE. An overview of cognitive impairment. Clin Geriatr Med 2018;34:505513.

39. Morley JE. The new geriatric giants. Clin Geriatr Med 217;33:xi-xii.

40. Morley JE. Rapid geriatric assessment: Secondary prevention to stop age-associated disability. Clin Geriatr Med 2017;33:431-440

41. Morley JE. Undernutrition in older adults. Fam Pract 2012;29(Suppl 1:i89-i93 . 